\title{
n2kanalysis: a framework for automated and reproducible statistics from long-term ecological monitoring
}

\section{Thierry Onkelinx}

Case study long-term monitoring

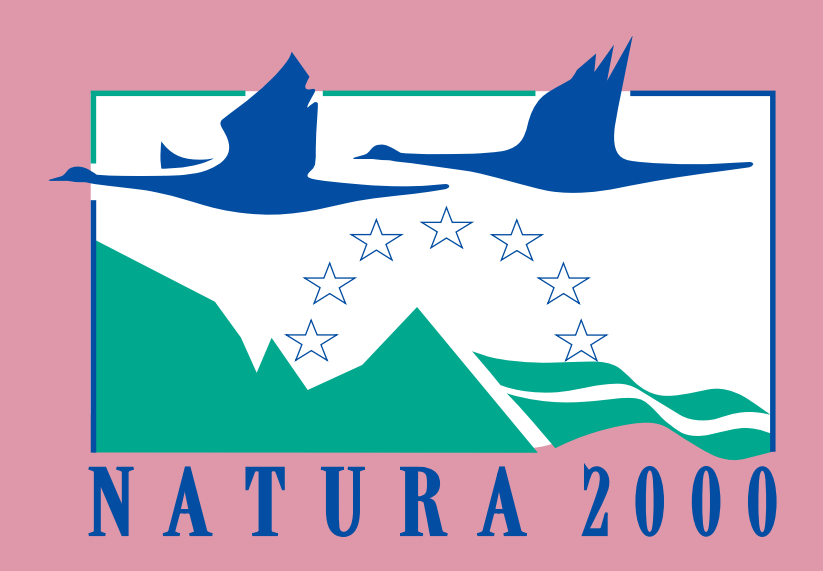

Natura 2000 (n2k) is a network of core sites breeding and resting sites for rare and threatened species

some rare natural habitat types

$18 \%$ of EU's land territory and $6 \%$ of its marine territory

member states must report every 6 year on status and trend over the last 24 year goal of $n 2 k a n a l y s i s:$ generate automated reproducible and traceable statistics

\section{Reproducible research}

\section{benefits}

repeating the analysis

- on same data yields the same results

- on new data yields comparable results

- allows for inspection

what is analysed and how

useful when doubts arise with third parties

$\checkmark$ downsides

- a bit harder to manage

- requires more storage

- stands or falls on version control

code

data

- software environment

\section{Schematic data flow}

\section{external data}

various formats
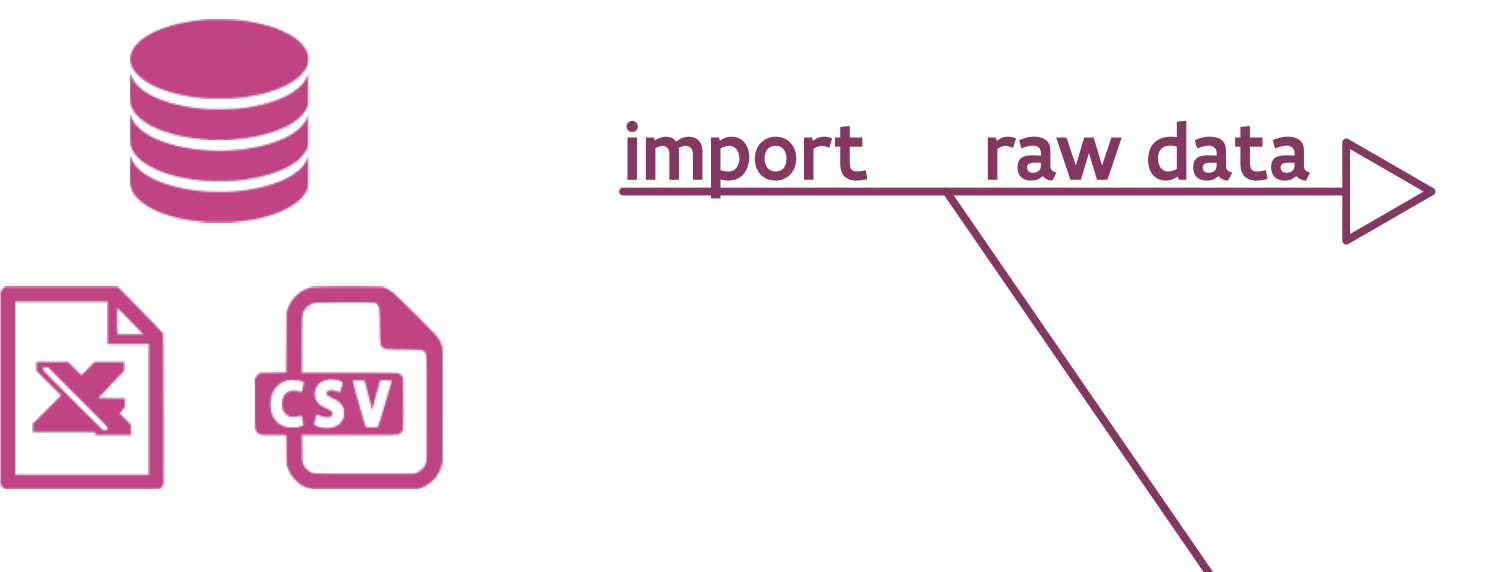

(1) $\begin{array}{r}\text { standardised } \\ \text { raw data }\end{array}$

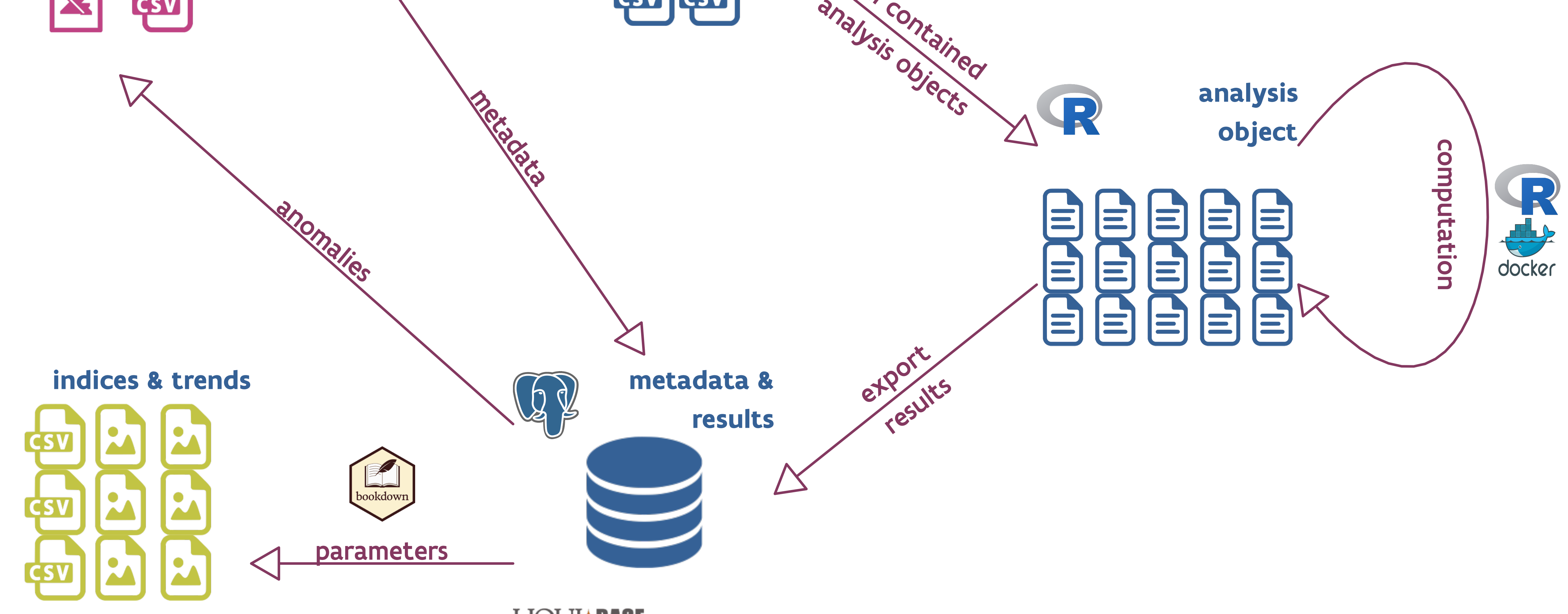

LIQUI১BASE

\section{Analysis object as cornerstone}

- S4 object n2kModel

- metadata

model type

- species group

- location group

- import date

- time range

- seed

file \& status fingerprint

- used software + version

- input for analysis

- data.frame

parent analysis when analysis depends on output of other analysis

- fitted model

\section{Anomalies?}

- extreme values according to the model

high (low) fitted values while low (high) observed values

extreme hyper parameters (e.g. random intercept with large $\sigma$ )

unstable imputations in case the analysis is based on multiple imputation

- might be due to

- typo in data

correct but strange observation

wrong model

- inspect only $n=10,20, \ldots$ extreme values

tackle the most influential errors first

- redo the analysis after fixing problems in the data

\section{Traceable research}

- retrace any parameter estimate to a specific analysis

- including data, metadata and environment

- solution: add file \& status fingerprints when communicating results

- fingerprint: SHA1 hashes

- file fingerprint: based on components which should never change in a specific analysis

stable metadata (model type, species group, location group, ...)

input data

doubles as file name for the analysis object

status fingerprint: based on file fingerprint + changing components

metadata (status, used software, ...) fitted model

\section{Data under version control}

analysis data

- private git repo

- even relative large monitoring schemes are doable 100 species, 1200 sites, 27 years, 6 month

- stable ordering of rows and columns is required

- Results

- PostgreSQL database only append data https://github.com/inbo/n2kresult

\section{Environment under version control}

- analyses run on virtual machine with Docker

- Docker image contains a fixed version of all required dependencies

- multiple versions of Docker image

- keep old versions for older analyses

- create new version when more recent software is required

\section{Pro tip: use nominal validation status}

- unchecked: default status for all records

- updated: records which have been altered

- good: scrutinized records which are correct and suitable for the original goal

- abnormal: scrutinized records which are correct but not suitable for the original goal

- rejected: scrutinized records which cannot be trusted

- anomaly: records which have not been scrutinized and flagged by an analysis as anomaly

Custom R packages under version control. Available at https://github.com/inbo

n2kanalysis

- generic package, used for every monitoring

- defines $\mathbf{S 4}$ classes + validation

- fits the analysis objects

- extracts model parameters and anomalies 'xyz' analysis

- 'xyz' stands for a specific monitoring scheme

- each monitoring scheme has its own package

- defines import of raw data and metadata

- defines how the analysis objects are created auxiliary packages

- n2khelper contains generic auxiliary functions - n2kupdate: export from R to database 